\title{
«Algunas raíces filosóficas del nazismo» de Aron Gurwitsch
}

\author{
INTRODUCCIÓN, TRADUCCIÓN Y NOTAS DE \\ MARI LUZ PINTOS PEÑARANDA \\ Universidad de Santiago de Compostela \\ (TRADUCCIÓN REALIZADA EN COLABORACIÓN \\ CON EVARISTO QUINTÁNS HERMIDA)
}

Recibido: 05-11-2009

Aprobado definitivamente: 14-11-2009

INTRODUCCIÓN AL TEXTO «ALGUNAS RAÍCES FILOSÓFICAS DEL NAZISMO»

EL ENSAYO QUE AQUí ESTAMOS PRESENTANDO traducido al castellano fue escrito por el filósofo Aron Gurwitsch. La versión original inglesa lleva por título «Some Philosophical Roots of Nazism» y forma parte del conjunto de manuscritos inéditos conservados en el Gurwitsch Nachlass del Archival Repository, Inc., del Center for Advanced Research in Phenomenology (CARP). El manuscrito está redactado a máquina. Tiene un total de 20 páginas, con unas treinta líneas en cada una de ellas. Hay algunas correcciones a mano, del propio autor; casi todas ellas son de carácter tipográfico.

En mi opinión, ciertas características de este ensayo añaden un valor extra al valor que ya tiene de por sí la reflexión sobre el nazismo que en él se ofrece:

1) Contrariamente a lo que suele creerse, no hay demasiados textos filosóficos sobre el nazismo; y menos todavía que estén escritos en el mismo momento de la segunda guerra mundial. Sin embargo, este ensayo de 
Gurwitsch es de 1942; ni había acabado la contienda ni todavía era conocido el fenómeno del holocausto.

2) Gurwitsch era un judío residente en Alemania cuando el nacional-socialismo subió al poder en 1933. Es por ello que su interés en reflexionar sobre el nazismo es, en primer término, un interés subjetivo y existencial, ya que sufrió en su propia historia personal y familiar las consecuencias de esta ideología y necesitaba buscar alguna razón para esta sinrazón. Este fondo de interés subjetivo, de ningún modo explicitado de forma abierta en el ensayo, infunde a éste todavía más intensidad dramática: debemos tener muy presente que, quien lo redacta, no estaba haciendo en ese momento una indagación más entre otras y sobre un asunto con respecto al que mediaba en él una actitud de indiferencia, sino una indagación que le cogía vitalmente muy de cerca.

3) Que esa locura de la ideología y actividades nazis tuviera su origen en Alemania quizás venía posibilitada por algunos de los componentes de la filosofía de esa zona cultural. Esto es lo que el autor pretende rastrear aquí, aun reconociendo, como hace, que el fenómeno del nacional-socialismo sólo puede ser explicado teniendo a la vista múltiples factores y de muy diverso tipo y no reduciéndose al filosófico.

4) Con independencia del nivel de profundidad que alcance el análisis gurwitscheano, y llegue a las conclusiones a las que llegue este análisis, el hecho es que tenemos ante nosotros un valioso ejercicio de autoanálisis llevado a cabo por un autor que se pregunta él mismo, como filósofo, sobre el asunto del nazismo; y que se pregunta no sólo como filósofo sin más, sino como filósofo que, criado en Alemania desde los cinco años y formado íntegramente en las Universidades alemanas, había hecho total inmersión en esa cultura y en esa filosofía, conociéndolas muy bien. El Gurwitsch filósofo reflexiona sobre el nazismo desde el seno de la filosofía alemana.

Por tanto, las preguntas que Gurwitsch dirige al nazismo están planteadas, por una parte, desde esa necesidad existencial que él tiene, como judío afectado de modo directo, de hallar alguna explicación a toda esa barbaridad e inconmensurable sufrimiento provocado por los nazis; pero, al mismo tiempo, por otra parte, son también preguntas planteadas desde ese interés de un filósofo que desea verificar objetivamente si el nazismo tuvo en parte su génesis en la propia filosofía, es decir, por lo menos «en cierta orientación de ella». Porque, de ser así, únicamente desenmascarando la perniciosidad a la que de algún modo propicia esta cierta orientación, estaríamos en disposición de intentar tomar distancia de ella y de no reproducir nunca más sus errores.

Aron Gurwitsch nació en Vilnius, Lituania, en 1901, en el seno de una familia judía de buena posición. En aquel momento, Lituania formaba parte del 
imperio ruso. Las persecuciones a los judíos (pogromos) que tuvieron lugar en Rusia en el siglo diecinueve y a comienzos del veinte, hicieron que la familia Gurwitsch se trasladase a Alemania en 1906. En este país inicia y completa todos los niveles de educación escolar. Cursó sus estudios universitarios en los años veinte en las Universidades de Berlín, Frankfurt y Gotinga. Durante esos años trabaja con destacados miembros de la nueva psicología de la Gestalt y con el neuropsiquiatra Kurt Goldstein y, a la vez, entra en contacto con Edmund Husserl y su fenomenología, pasando a ser uno de sus discípulos directos más fieles. Desde su contacto con Husserl, toda su investigación y su docencia estarán orientadas desde la fenomenología. La tesis doctoral que presenta en Gotinga en $1928^{1}$ expresa a la perfección la peculiar orientación del fenomenólogo Aron Gurwitsch, ya que lo que hace en ese trabajo, centrándose en la psicología gestaltista y en la filosofía fenomenológica husserliana, es lo que después hará durante los años que le siguen y durante toda la década de los años treinta: Gurwitsch intentaba buscar puntos de convergencia entre la filosofía (fenomenológica) y otras ciencias en las que a él le parecía que en ese momento estaba surgiendo una nueva orientación más hacia lo vivido y lo concreto (la psicología, la psiquiatría, la lingüística, la sociología, la antropología, etc.). En su tesis de habilitación, redactada en la Universidad de Berlín y finalizada en $1933,{ }^{2}$ hace una fenomenológica clarificación -como él mismo dice- de algunos fundamentales conceptos de Max Weber y del fenómeno del humano «Miteinandersein» (ser-unos-con-otros). En esos años, su compromiso con la causa judía está manifiesto en varios escritos breves publicados para la comunidad judía. Y es muy probable que la importancia que tenía para él la causa judía -concretizada en la mala convivencia entre los judíos y los habitantes del país europeo en donde estuvieran asentados- llevase a Gurwitsch a interesarse por el asunto de la intersubjetividad y de la inevitable interrelación, buena o mala, que los humanos mantenemos entre nosotros.

En 1933 se exilia a Francia y desde 1934 hasta 1939 es encargado de curso en el Institut d’Histoire des Sciences de la Universidad de la Sorbona. Los

1 Phänomenologie der Thematik und des reinen Ich. Studien über Beziehungen von Gestalttheorie und Phänomenologie, en Psychologische Forschung, Bd. 12, p. 282; o cf., en su versión inglesa, Phenomenology of Thematics and of the Pure Ego: Studies of the Relation between Gestalt Theory and Phenomenology, en Aron Gurwitsch Studies in Phenomenology and Psychology, Evanston: Northwestern University Press, 1966, pp. 176s; traducción hecha por Fred Kersten.

2 Die mitmenschlichen Begegnungen in der Milieuwelt, Berlin: Walter de Gruyter, 1977; edición de Alexandre Métraux. En versión inglesa: Human Encounters in the Social World. Pittsburgh: Duquesne University Press, 1979; traducido por Fred Kersten. 
cursos que ahí dicta son, en Francia, la primera introducción sistemática a la fenomenología y una de las más fieles y profundas exposiciones de ella. ${ }^{3}$

En 1940, cuando las tropas alemanas invaden Francia y él se ve forzado a un tercer exilio, esta vez a los Estados Unidos, se hallaba trabajando -o ya este trabajo estaba concluido; no se puede precisar con exactitud- en «Sobre el nihilismo de nuestro tiempo», ${ }^{4}$ un ensayo en el que aborda el fenómeno del nazismo, sus técnicas embaucadoras y el consiguiente seguimiento de la gente. Gurwitsch entra en su nuevo país de acogida con este ensayo, si bien las dificultades para encontrar editor le hacen esperar hasta 1945 para verlo publicado. Como su título indica, este ensayo no está centrado en al nazismo, pero, sin embargo, en su desarrollo interno sí se refiere a él como un fenómeno propio de la sociedad occidental de «nuestro tiempo». Sin duda basándose en la propia descripción husserliana de nuestra cultura y sociedad, en la descripción que hace Gurwitsch de esta sociedad, (a) el «naturalismo» la impregna totalmente, (b) el nihilismo es presupuesto y base del «naturalismo» y (c) el nazismo es una consecuencia más de esta atmósfera cultural. Con otras palabras, el nihilismo es el presupuesto y la base del «naturalismo» de nuestra época; y, a su vez, el movimiento totalitario nazi es la culminación del nihilismo.

Gurwitsch usa el término «nihilismo» para referirse a aquella interpretación del ser humano que consiste en tomarlo únicamente por su lado biológico, es decir, tan sólo como un ser con necesidades e impulsos vitales ligados a su biología y a costa de desatender el hecho de que, además de ser «vital», el ser humano es también «racional» y, por tanto, con la capacidad y la inexcusable responsabilidad de un comportamiento ético. Cuando se abandona la idea del ser humano como ser racional, con toda la carga de responsabilidad moral que ello implica, la «razón» pasa a ser tenida tan sólo -o principalmente- como aquel «instrumento» inteligente que puede ser puesto al servicio de los deseos y necesidades particulares, del tipo que fuere. La consecuencia de haber dejado de interpretar al ser humano como un ser «racional» y, por tanto, de haber dejado de verlo como un ser con la capacidad de, y con la inexcusable obligación moral para, buscar y querer la verdad, es la de que se abandona el ideal (racio-

3 En la actualidad, tan sólo dos de estos cursos están publicados. Se trata del curso impartido en 1933-1934, «Quelques aspects et developpements de la Psychologie de la Forme», Journal de Psychologie Normal et Pathologique vol. 33 (1936) 413-470, y del curso impartido en 1937-38, Esquisse de la phénoménologie constitutive, Paris: Vrin, 2002, 418 págs. (el editor es José Huertas-Jourda y la introducción es de Lester Embree).

4 «El nihilismo de nuestro tiempo» está publicado en castellano en Agora. Papeles de Filosofía, vol. 22, 2 (2003) 157-192. La traducción, de la que soy autora, se hizo sobre el manuscrito original, redactado originalmente en francés - «Sur le nihilisme de notre temps»-y en la mencionada revista va precedida por una «Introducción al texto» escrita por Lester Embree. 
nal) de aspiración a un mundo mejor para todos y la de que este ideal pasa a ser sustituido por el de la mera utilidad y por el de la satisfacción inmediata de deseos y necesidades particulares, tanto por parte de individuos como por parte de grupos. Sin duda, señala Gurwitsch, esta interpretación científica de corte objetivista-naturalista acerca de los humanos y del resto de los seres del planeta proporciona un excelente apoyo para aquellos dirigentes políticos y grupos de poder que están interesados en movernos en pro de sus particulares intereses. La concepción nihilista sustituye la idea de verdad por la de mera «opinión». No hay una verdad, sino que únicamente hay «opiniones»; opiniones que serán o no serán verdaderas tan sólo en la medida del valor utilitario y funcional que ellas comporten. No hay ninguna verdad válida para todos y siempre (como, por ejemplo, la de querer $-\mathrm{y}$, de hecho trabajar por- hacer realidad un mundo mejor para todos) a la que cada individuo o grupo deba adaptar su pensamiento y forma de vida, sino que más bien es al revés: tendrá valor de verdad tan sólo aquello que es útil; y sólo será útil aquello que se adapte a los intereses y beneficios particulares de cada individuo o grupo. Y esto, dice Gurwitsch, es precisamente lo que sucedió con el totalitarismo nazi: el grupo que tiene el poder somete a los demás y, para ello, justifica todo tipo de medios empleados bajo la consideración de superioridad y de que esta superioridad es una «ley de la naturaleza» que, por tanto, todos debemos acatar ciegamente. Según él, desde la Ilustración hasta nuestros días, aquello que inicialmente se entendía por «progreso» ha finalizado en un proyecto ajeno no sólo a la Ilustración sino a todos los siglos de la historia occidental: el proyecto nazi del exterminio de naciones enteras y de la reducción de otras a la esclavitud.

Después de haber redactado este ensayo sobre el nihilismo, y ya en los Estados Unidos, durante los años en los que tiene lugar la guerra Gurwitsch continúa analizando el fenómeno del nazismo y dejando testimonio escrito de su análisis bajo dos estilos muy diferentes: uno es el epistolar, en el ámbito privado; otro es el del ensayo filosófico. En efecto, durante 1941, mantiene correspondencia epistolar con Dorion Cairns, ${ }^{5}$ también como él discípulo directo de Husserl y al que ya había conocido en Alemania años antes. En mi opinión, el debate que surge en esas cartas en torno a distintos aspectos del nazismo con respecto a los que no se ponen de acuerdo entre ellos dos, sirvió de motivo a Gurwitsch para poner sus hipótesis por escrito. El resultado es su ensayo sobre «Algunas raíces filosóficas del nazismo». Sin embargo, una vez escrito, probablemente no hizo ningún intento serio por publicarlo $\mathrm{y}$, finalmente, ha permanecido inédito hasta ahora.

5 «Two Husserlian Discuss Nazism: Letters between Dorion Cairns and Aron Gurwitsch in 1941», Husserl Studies 8 (1991) 77-105; editor Lester Embree. 
No es difícil imaginarse que en 1942, cuando escribe este ensayo, Gurwitsch preferiría ser prudente y no hacer públicas sus ideas sobre la cultura filosófica alemana. En primer lugar, nadie podía estar seguro en ese momento de quiénes serían los vencedores en esa guerra que estaba teniendo lugar a nivel mundial y, por consiguiente, todo aconsejaba ser muy prudente. En segundo lugar, podemos suponer que Gurwitsch no querría convertirse en un ciudadano incómodo para el país que lo había acogido, ni tampoco destacar en sentido negativo; su idea sobre la filosofía alemana y las opiniones que vierte en este ensayo quizás no fuesen del agrado de todos los estadounidenses y, en aquellos años difíciles, en los que Gurwitsch sobrevivía económicamente a duras penas y en puestos de trabajo precarios, mejor era ser cuidadoso. En tercer lugar, por entonces ya contaba él con una mala experiencia: la de las dificultades que estaba encontrando para publicar su ensayo sobre el nihilismo de nuestra cultura actual -ensayo que, por cierto, una vez publicado en 1945 le ocasionó la pérdida de por lo menos tres puestos de trabajo. Lo que dice Jürgen Habermas para explicar el traslado que Adorno y de Horkheimer hacen en 1940-1941 desde la Universidad de Columbia, en New York, a California, quizás puede ser también aplicado a Aron Gurwitsch en relación a su decisión de mantener un perfil bajo en cuanto a su posición sobre las raíces filosóficas del nazismo. Señala Habermas que Horkheimer se fue a California porque «tenía temores políticos de que, incluso en América, la tendencia fascista se hiciera tan fuerte que peligrara el Instituto como tal». ${ }^{6}$ Esto significa que este temor estaba en el ambiente.

Vayamos ahora al ensayo sobre «Algunas raíces filosóficas del nazismo». Contiene tan sólo una división explicitada; pero da la impresión de que ésta es más una división señalada por Gurwitsch para él mismo -con esa provisionalidad de cuando uno está redactando- que para aparecer de esta manera en una publicación, ya que no es normal que aparezca una división sola y con sólo una palabra: «Absoluto». De modo que, prescindiendo de esta marca, se aprecian en el ensayo hasta cinco partes bien diferenciadas:

En la primera parte (párrafos 1-3), Gurwitsch inicia el texto indicando que el movimiento nazi no puede explicarse tan sólo como un fenómeno político; hay en él múltiples factores que se dieron al unísono y que hicieron que surgiera y que tuviera éxito. Gurwitsch anuncia que se centrará en estas cuestiones: 1) ¿Por qué esa falta de resistencia a la ideología nazi por parte de las clases intelectuales en Alemania? 2) ¿Hay elementos en la tradición alemana nacional que pueden arrojar alguna luz sobre esta extraña falta de resistencia? ¿Cómo pudo crecer esta ideología, con todas las creencias que en ella van implicadas, en Alemania? 3) Y, ¿era esta ideología tan sólo una herramienta política o era una

6 Jürgen Habermas, Perfiles filosófico-politicos, Madrid: Taurus, 1979, p. 247. 
nueva verdad filosófica - una verdad filosófica que terminaría trayendo consigo desmoralización y esclavitud?

En la segunda parte (párrafos 4-11), Gurwitsch plantea su hipótesis: sí hubo ciertos aspectos en la tradición filosófica alemana que favorecieron la aparición del nacional-socialismo. Estos aspectos pertenecen al movimiento idealista florecido en el Romanticismo alemán que va entre 1794 (con la publicación de la Wissenschaftslehre) y 1831 (con la muerte de Hegel). La filosofía del idealismo alemán se separa de la filosofía universal de la Europa occidental, trazando de este modo un corte con la gran tradición filosófica de los siglos XVII y XVIII -con aquella filosofía que estaba estrechamente relacionada con la ciencia: el vehículo del conocimiento era el sentido común o el método científico y el razonamiento, por el que se alcanza el conocimiento limitado y relativo de la ciencia; al cortar intencionalmente con esto, el idealismo se va a apoyar en la «razón especulativa» para alcanzar el Absoluto. Según Gurwitsch, el modo idealista de interpretar el mundo es el panteísmo: la realidad y todos los hechos como tal quedan deificados y sólo son vistos en su conexión con el Absoluto. La hipótesis gurwitscheana es doble: a) el panteísmo está en la raíz de los fenómenos políticos y morales del nazismo; b) este panteísmo está conectado con la deificación del Estado en Hegel -el Estado no está en realidad para servir al bienestar de sus miembros, sino que es la encarnación de la voluntad divina. Gurwitsch describe esta forma de interpretar la realidad y de interpretar el pensamiento de mitología, y no de filosofía; una mitología que resultó ser extremadamente eficaz para generar la mentalidad nazi. Se sustituyen los términos hegelianos por los pseudobiológicos (los que se dan con el «nihilismo alemán»), y el resultado es la ideología nacional-socialista.

En la tercera parte (párrafos 12-14), comienza Gurwitsch afirmando que esto que vale para la historia de la filosofía, vale también para la historia política, la cual es expresión de la progresiva automanifestación de la Razón Absoluta. El panteísmo de Hegel, permite consagrar, con el Führer, el «orden establecido de las cosas» y el conformismo que de ahí resulta. «La filosofía de la historia de Hegel es la base metafísica y la justificación de la Gleichschaltung expresada en términos metafísicos», dice.

La cuarta parte (párrafos 15-21) está dedicada a Fichte, ya que fue él quien proclamó en 1807-1808 que a Alemania le estaba llegando su turno en el proceso de gradual despliegue por el que va pasando el Weltgeist. Fichte había hablado de la oposición de «Alemania» frente al «mundo europeo occidental»y, al insistir y profundizar en esta oposición, se obtienen también las del «Geist» frente al «esprit», la del «organicismo» frente al «mecanicismo», la del «luchar para entender las cosas desde dentro (Gëmut)» frente al «calcular acerca de lo externo de las cosas», la de «el alemán hace las cosas por su propio interés» frente al «utilitarismo occidental». El nacionalismo alemán es antiliberal desde 
su comienzo debido a que se basa en esa profundidad buscada por Fichte (la aboriginalidad) y, por tanto, el antiliberalismo es, según Gurwitsch, la piedra angular del sistema nacional-socialista.

La quinta parte (párrafos 22-final) muestra la hipótesis central gurwitscheana de que el Idealismo alemán es una de las raíces del nazismo, aunque también haya otras que lo expliquen. Gurwitsch encuentra un modo de probar esta hipótesis: mucho antes de hacer aparición el fenómeno nazi, ya hubo quien, basándose únicamente en lo que le parecía que era la esencia de la filosofía idealista alemana, fue capaz de prever, sin equivocarse, la destrucción que surgiría más pronto o más tarde desde Alemania «para llenar el mundo de espanto y admiración». Se trata del historiador francés Hippolyte Taine, quien agudamente anunció por anticipado, en 1834, la aparición de este fenómeno.

En los años siguientes a la redacción de este ensayo, Gurwitsch intercambia algún que otro comentario sobre el fenómeno del nazismo en la correspondencia que mantiene con Alfred Schutz ${ }^{7}$ y en la correspondencia con Eric Vögelin. Después de finalizada la guerra, permanece en los Estados Unidos, junto a su esposa Alice, si bien acudiendo continuamente a Europa y a otras zonas para dar conferencias en distintas Universidades. En el año 1959, y hasta su fallecimiento, fue contratado como profesor de filosofía por la New School for Social Research, en New York. En esta etapa, que duró doce años, se consagró como el gran divulgador de la fenomenología en los Estados Unidos y, como señala Lester Embree, ${ }^{8}$ buena parte de la fenomenología que se hizo en ese país desde los años 70 tiene su huella, y ésta todavía permanece muy viva hasta hoy. De entre todos los textos publicados durante esos años, sin duda merece ser destacado el único que por el momento está traducido al castellano, El campo de la conciencia: un análisis fenomenológico, ${ }^{9}$ y por el que es más conocido en Europa.

Con esta traducción de «Algunas raíces filosóficas del nazismo» que aquí estamos publicando, esperamos contribuir a la recuperación en Europa de la filosofía de este pensador a quien, desafortunadamente, todavía hoy no conocemos lo suficiente debido a su exilio en los Estados Unidos -hasta hace poco tiempo, los europeos teníamos poco acceso a los textos filosóficos publicados

7 Alfred Schütz, Aron Gurwitsch: Briefwechsel, 1939-1959, München: W. Fink Verlag, 1985; editor Richard Gratoff. Versión inglesa: Philosophers in Exile: The Correspondence of Alfred Schutz and Aron Gurwitsch, 1939-1959, Bloomington \& Indianapolis: Indiana University Press, 1989; traducción hecha por Claude Evans.

$8 C f$. Lester Embree, «Biographical Sketch of Aron Gurwitsch», texto online en The Aron Gurwitsch Page, en www.gurwitsch.net, y $c f$. también el texto de Embree referido in supra, en nota 4.

9 El campo de la conciencia, Madrid, Alianza, 1979; traducción desde el inglés por Jorge García-Gómez. 
allí. Pretendemos, además, que la publicación de este ensayo contribuya a sacar a la luz su faceta de pensador menos conocida: la de su reflexión comprometida con las circunstancias de su tiempo. 
